\title{
Maillard Reaction Products in Different Types of Brewing Malt
}

Michael Hellwig, Thomas Henle

Chair of Food Chemistry, Technische Universität Dresden, D-01062 Dresden, Germany

Corresponding author:

T. Henle

Tel.: +49-351-463-34647

Fax: +49-351-463-34138

Email: Thomas.Henle@tu-dresden.de 
Table S1. Malts Analyzed in this Study and Concentrations of Maillard Reaction Products in Individual Samples (Data are means \pm S.D., $n=2-3$ ).

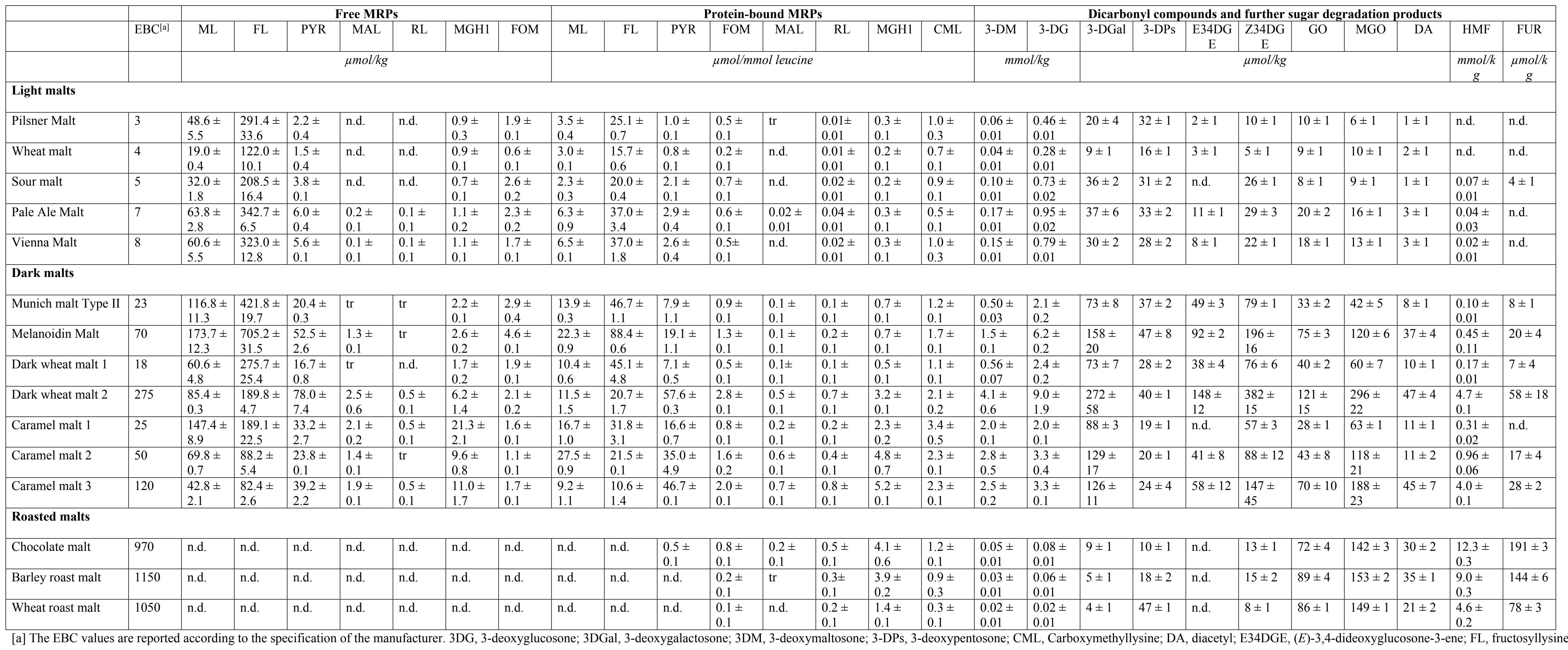

[a] The EBC values are reported according to the specification of the manufacturer. 3DG, 3-deoxyglucosone; 3DGal, 3-deoxygalactosone; 3DM, 3-deoxymalttosone; 3-DPs, 3-deoxypentosone; CML, Carboxymethyllysine; DA, diacetyl; E34DGE, (E)-3,4-dideoxyglucosone-3-en
FOM, formyline; FUR, furfural; GO, glyoxal; HMF, 5-hydroxymethylfurfural; MAL, maltosine; MG-H1, methylglyoxal-derived hydroimidazolone 1; MGO, methylglyoxal; ML, maltulosyllysine; PYR, pyrraline; RL, rhamnolysine; Z34DGE, (Z)-3,4-dideoxyglucosone-3-ene. 
Table S2. Transitions Recorded during MRM Measurement of MRPs in Malt.[a]

\begin{tabular}{|c|c|c|c|c|c|}
\hline Compound & $\begin{array}{l}\text { Precursor } \\
\text { ion }[\mathrm{m} / \mathrm{z}]\end{array}$ & $\begin{array}{l}\text { Product } \\
\text { ion } \\
{[\mathrm{m} / \mathrm{z}]}\end{array}$ & $\begin{array}{l}\text { Fragmentor } \\
\text { voltage [V] }\end{array}$ & $\begin{array}{l}\text { Collision } \\
\text { energy } \\
{[\mathrm{eV}]}\end{array}$ & $\mathrm{Q} / \mathrm{q}^{[\mathrm{b}]}$ \\
\hline \multirow[t]{2}{*}{ Maltulosyllysine } & 471 & 128 & 140 & 20 & $q$ \\
\hline & 471 & 225 & 140 & 20 & Q \\
\hline \multirow[t]{2}{*}{ 3-DG-H } & 319 & 204 & 100 & 20 & Q \\
\hline & 319 & 116 & 100 & 20 & $\mathrm{q}$ \\
\hline \multirow[t]{2}{*}{ Fructosyllysine } & 309 & 84 & 120 & 30 & Q \\
\hline & 309 & 225 & 120 & 10 & $\mathrm{q}$ \\
\hline \multirow[t]{2}{*}{ Pyrraline } & 255 & 148 & 80 & 20 & $\mathrm{q}$ \\
\hline & 255 & 175 & 80 & 10 & Q \\
\hline \multirow[t]{2}{*}{ Maltosine } & 255 & 84 & 120 & 20 & Q \\
\hline & 255 & 126 & 120 & 10 & $q$ \\
\hline \multirow[t]{2}{*}{ Rhamnolysine } & 239 & 175 & 100 & 12 & $\mathrm{q}$ \\
\hline & 239 & 148 & 100 & 24 & Q \\
\hline \multirow[t]{2}{*}{ MG-H1 } & 229 & 114 & 120 & 10 & Q \\
\hline & 229 & 166 & 120 & 10 & $q$ \\
\hline \multirow[t]{2}{*}{ Formyline } & 225 & 134 & 80 & 20 & Q \\
\hline & 225 & 161 & 80 & 10 & $q$ \\
\hline
\end{tabular}

[a] general conditions-dwell time, $70 \mathrm{~ms}$; positive mode. [b] Q, transition used for quantitation; q, transition used for the confirmation of the presence of the analyte. 
Table S3. Transitions Recorded during MRM Measurement of Quinoxalines in Malt. ${ }^{[a]}$

\begin{tabular}{|c|c|c|c|c|c|c|}
\hline $\begin{array}{l}\text { Time frame } \\
{[\mathrm{min}]}\end{array}$ & Compound & $\begin{array}{l}\text { Precursor } \\
\text { ion }[m / z]\end{array}$ & $\begin{array}{l}\text { Product } \\
\text { ion }[\mathrm{m} / \mathrm{z}]\end{array}$ & $\begin{array}{l}\text { Fragmentor } \\
\text { voltage }[\mathrm{V}]\end{array}$ & $\begin{array}{l}\text { Collision } \\
\text { energy } \\
{[\mathrm{eV}]}\end{array}$ & $\mathrm{Q} / \mathrm{q}^{[\mathrm{b}]}$ \\
\hline \multirow[t]{2}{*}{$3-5$} & 3-deoxymaltosone & 397 & 235 & 100 & 10 & Q \\
\hline & & 397 & 217 & 100 & 20 & $q$ \\
\hline \multirow[t]{4}{*}{$5-8.5$} & 3-deoxyglucosone & 235 & 199 & 80 & 10 & $\mathrm{Q}$ \\
\hline & & 235 & 171 & 80 & 20 & $\mathrm{q}$ \\
\hline & 3-deoxygalactosone & 235 & 217 & 80 & 10 & $\mathrm{q}$ \\
\hline & & 235 & 145 & 80 & 20 & $\mathrm{Q}$ \\
\hline \multirow[t]{2}{*}{$8.5-13.5$} & 3-deoxypentosone & 205 & 187 & 120 & 10 & $\mathrm{q}$ \\
\hline & & 205 & 145 & 120 & 20 & Q \\
\hline \multirow[t]{10}{*}{$13.5-24$} & 3-deoxyrhamnosone & 219 & 201 & 80 & 12 & $q$ \\
\hline & & 219 & 145 & 120 & 18 & Q \\
\hline & $\begin{array}{l}\text { 3,4-dideoxy- } \\
\text { glucosone-3-ene } e^{[b]}\end{array}$ & 217 & 169 & 120 & 12 & Q \\
\hline & & 217 & 145 & 120 & 12 & $q$ \\
\hline & glyoxal & 131 & 104 & 80 & 20 & $\mathrm{q}$ \\
\hline & & 131 & 77 & 80 & 30 & $\mathrm{Q}$ \\
\hline & Methylglyoxal & 145 & 118 & 100 & 20 & $q$ \\
\hline & & 145 & 77 & 100 & 30 & $\mathrm{Q}$ \\
\hline & diacetyl & 159 & 118 & 100 & 20 & $\mathrm{Q}$ \\
\hline & & 159 & 77 & 100 & 40 & $q$ \\
\hline
\end{tabular}

[a] General conditions: dwell time, $250 \mathrm{~ms}$ (3-13.5 min), $120 \mathrm{~ms}$ (13.5-24 min). [b] The same conditions proved optimal for both isomers. 


\begin{tabular}{|c|c|c|c|c|c|c|c|c|c|c|c|c|c|c|c|c|c|}
\hline & & ML & PYR & $\mathbf{R L}$ & MGH1 & FOM & CML & 3DM & 3DG & 3DGal & GO & E34DGE & Z34DGE & DA & MGO & HMF & FUR \\
\hline Color & $\begin{array}{l}\mathrm{r}_{\mathrm{s}} \\
\mathrm{P} \\
\mathrm{N} \\
\end{array}$ & $\begin{array}{c}, 762 \\
, 004 \\
12 \\
\end{array}$ & & $\begin{array}{r}, 853 \\
, 000 \\
15 \\
\end{array}$ & $\begin{array}{c}835 \\
, 000 \\
15 \\
\end{array}$ & & & & & & $\begin{array}{l}, 932 \\
, 000 \\
15 \\
\end{array}$ & $\begin{array}{c}964 \\
, 000 \\
10 \\
\end{array}$ & & $\begin{array}{l}, 882 \\
, 000 \\
15 \\
\end{array}$ & $\begin{array}{r}, 936 \\
, 000 \\
, 15 \\
\end{array}$ & $\begin{array}{l}, 940 \\
, 000 \\
13 \\
\end{array}$ & $\begin{array}{c}9600 \\
, 000 \\
10 \\
\end{array}$ \\
\hline ML & $\begin{array}{l}r_{\mathrm{S}} \\
P \\
\mathrm{~N}\end{array}$ & & $\begin{array}{c}, 769 \\
, 003 \\
12\end{array}$ & $\begin{array}{c}, 733 \\
, 007 \\
12\end{array}$ & $\begin{array}{c}, 802 \\
, 002 \\
12\end{array}$ & & & $\begin{array}{c}, 790 \\
, 002 \\
12\end{array}$ & $\begin{array}{c}, 797 \\
, 002 \\
12\end{array}$ & $\begin{array}{l}, 797 \\
, 002 \\
12\end{array}$ & $\begin{array}{c}, 776 \\
, 003 \\
12\end{array}$ & & $\begin{array}{c}, 741 \\
, 006 \\
12\end{array}$ & $\begin{array}{c}, 755 \\
, 005 \\
12\end{array}$ & $\begin{array}{c}, 748 \\
, 005 \\
12\end{array}$ & & \\
\hline PYR & $\begin{array}{l}\mathrm{r}_{\mathrm{S}} \\
P \\
\mathrm{~N}\end{array}$ & & & & & $\begin{array}{c}, 841 \\
, 000 \\
13\end{array}$ & $\begin{array}{c}, 736 \\
, 004 \\
13\end{array}$ & $\begin{array}{c}, 978 \\
, 000 \\
13\end{array}$ & $\begin{array}{c}.956 \\
.000 \\
13\end{array}$ & $\begin{array}{c}.967 \\
, 000 \\
13\end{array}$ & & $\begin{array}{c}, 927 \\
, 000 \\
10\end{array}$ & $\begin{array}{c}, 945 \\
, 000 \\
13\end{array}$ & $\begin{array}{l}, 698 \\
, 008 \\
13\end{array}$ & & & \\
\hline MAL & $\begin{array}{l}\mathrm{r}_{\mathrm{S}} \\
\mathrm{P} \\
\mathrm{N} \\
\end{array}$ & & & $\begin{array}{c}900 \\
, 001 \\
9 \\
\end{array}$ & $\begin{array}{c}, 933 \\
, 000 \\
9 \\
\end{array}$ & & $\begin{array}{c}, 917 \\
, 001 \\
9 \\
\end{array}$ & & & & & & & & & & \\
\hline RL & $\begin{array}{l}\mathrm{r}_{\mathrm{S}} \\
\mathrm{P} \\
\mathrm{N} \\
\end{array}$ & & & & $\begin{array}{c}957 \\
, 000 \\
15 \\
\end{array}$ & & & & & & $\begin{array}{c}805 \\
, 000 \\
15 \\
\end{array}$ & $\begin{array}{c}, 912 \\
, 000 \\
10 \\
\end{array}$ & & $\begin{array}{c}886 \\
, 000 \\
15 \\
\end{array}$ & $\begin{array}{c}, 923 \\
, 000 \\
15 \\
\end{array}$ & $\begin{array}{c}, 853 \\
, 000 \\
13 \\
\end{array}$ & \\
\hline MGH1 & $\begin{array}{l}\mathrm{r}_{\mathrm{S}} \\
P \\
\mathrm{~N}\end{array}$ & & & & & & & & & & $\begin{array}{c}, 774 \\
, 001 \\
15 \\
\end{array}$ & $\begin{array}{c}.851 \\
, 002 \\
10\end{array}$ & & $\begin{array}{c}.842 \\
, 000 \\
15 \\
\end{array}$ & $\begin{array}{c}.870 \\
, 000 \\
15 \\
\end{array}$ & $\begin{array}{c}, 817 \\
, 001 \\
13 \\
\end{array}$ & \\
\hline FOM & $\begin{array}{l}\mathrm{r}_{\mathrm{S}} \\
\mathrm{P} \\
\mathrm{N}\end{array}$ & & & & & & $\begin{array}{c}889 \\
.000 \\
15\end{array}$ & $\begin{array}{c}, 889 \\
, 000 \\
15\end{array}$ & $\begin{array}{c}, 854 \\
.000 \\
15\end{array}$ & $\begin{array}{c}.875 \\
, 000 \\
15\end{array}$ & & $\begin{array}{c}.915 \\
, 000 \\
10\end{array}$ & $\begin{array}{c}.882 \\
, 000 \\
15\end{array}$ & & & & \\
\hline CML & $\begin{array}{l}r_{\mathrm{S}} \\
P \\
\mathrm{~N}\end{array}$ & & & & & & & $\begin{array}{c}, 814 \\
, 000 \\
15\end{array}$ & $\begin{array}{c}, 729 \\
, 002 \\
15\end{array}$ & $\begin{array}{c}, 779 \\
, 001 \\
15\end{array}$ & & & $\begin{array}{c}, 761 \\
, 001 \\
15\end{array}$ & & & & \\
\hline 3DM & $\begin{array}{l}\mathrm{r}_{\mathrm{S}} \\
\mathrm{P} \\
\mathrm{N}\end{array}$ & & & & & & & & $\begin{array}{c}, 957 \\
, 000 \\
15\end{array}$ & $\begin{array}{c}, 971 \\
, 000 \\
15\end{array}$ & & $\begin{array}{c}, 879 \\
, 001 \\
10\end{array}$ & $\begin{array}{c}.918 \\
, 000 \\
15\end{array}$ & & & & \\
\hline 3DG & $\begin{array}{l}r_{S} \\
P \\
N\end{array}$ & & & & & & & & & $\begin{array}{c}, 986 \\
, 000 \\
15\end{array}$ & & $\begin{array}{c}, 927 \\
, 000 \\
10\end{array}$ & $\begin{array}{c}, 946 \\
, 000 \\
15\end{array}$ & & & & \\
\hline 3DGal & $\begin{array}{l}\mathrm{r}_{\mathrm{S}} \\
P \\
\mathrm{~N}\end{array}$ & & & & & & & & & & & $\begin{array}{c}, 927 \\
, 000 \\
10\end{array}$ & $\begin{array}{l}, 939 \\
, 000 \\
15 \\
15\end{array}$ & & & & \\
\hline GO & $\begin{array}{l}\mathrm{r}_{\mathrm{S}} \\
\mathrm{P} \\
\mathrm{N} \\
\end{array}$ & & & & & & & & & & & $\begin{array}{c}, 952 \\
, 000 \\
10 \\
\end{array}$ & & $\begin{array}{r}, 918 \\
, 000 \\
15 \\
\end{array}$ & $\begin{array}{r}, 939 \\
, 000 \\
15 \\
\end{array}$ & $\begin{array}{c}, 879 \\
, 000 \\
13 \\
\end{array}$ & $\begin{array}{c}.815 \\
, 004 \\
10 \\
\end{array}$ \\
\hline E34DGE & $\begin{array}{l}\mathrm{r}_{\mathrm{s}} \\
\mathrm{P} \\
\mathrm{N}\end{array}$ & & & & & & & & & & & & $\begin{array}{c}, 976 \\
, 000 \\
10\end{array}$ & $\begin{array}{c}, 939 \\
, 000 \\
10\end{array}$ & $\begin{array}{c}.952 \\
, 000 \\
10\end{array}$ & & \\
\hline DA & $\begin{array}{l}\mathrm{r}_{\mathrm{S}} \\
P \\
\mathrm{~N}\end{array}$ & & & & & & & & & & & & & & $\begin{array}{c}, 964 \\
, 000 \\
15 \\
\end{array}$ & $\begin{array}{c}, 819 \\
, 001 \\
13 \\
\end{array}$ & \\
\hline MGO & $\begin{array}{l}\mathrm{r}_{\mathrm{S}} \\
\mathrm{P} \\
\mathrm{N}\end{array}$ & & & & & & & & & & & & & & & $\begin{array}{c}, 896 \\
, 000 \\
13\end{array}$ & $\begin{array}{c}, 778 \\
, 008 \\
10\end{array}$ \\
\hline HMF & $\begin{array}{l}\mathrm{r}_{\mathrm{S}} \\
P \\
\mathrm{~N}\end{array}$ & & & & & & & & & & & & & & & & $\begin{array}{c}, 973 \\
, 000 \\
10\end{array}$ \\
\hline
\end{tabular}

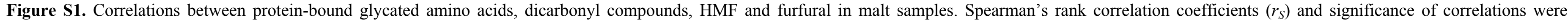

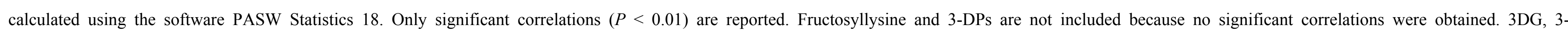

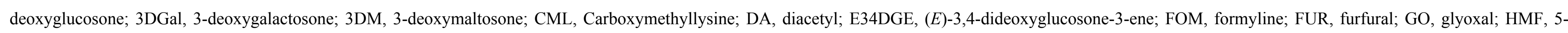

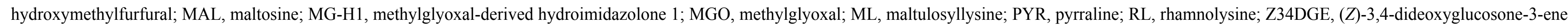




\begin{tabular}{|c|c|c|c|c|c|c|c|c|}
\hline & & & \multicolumn{6}{|c|}{ Protein-bound glycated amino acids } \\
\hline & & & ML & FL & PYR & MAL & MGH1 & FOM \\
\hline \multirow{6}{*}{ 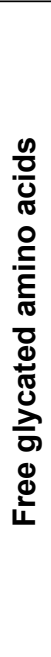 } & ML & $\begin{array}{l}r_{S} \\
P \\
N\end{array}$ & $\begin{array}{r}, 848 \\
, 000 \\
12\end{array}$ & & & & & \\
\hline & FL & $\begin{array}{l}r_{S} \\
P \\
N\end{array}$ & & $\begin{array}{r}, 846 \\
, 001 \\
12\end{array}$ & & & & \\
\hline & PYR & $\begin{array}{l}r_{S} \\
P \\
N\end{array}$ & & & $\begin{array}{r}, 965 \\
, 000 \\
12\end{array}$ & & & \\
\hline & MAL & $\begin{array}{l}\mathrm{r}_{\mathrm{S}} \\
P \\
\mathrm{~N}\end{array}$ & & & & $\begin{array}{r}, 764 \\
, 046 \\
7\end{array}$ & & \\
\hline & MGH1 & $\begin{array}{l}r_{\mathrm{S}} \\
P \\
\mathrm{~N} \\
\end{array}$ & & & & & $\begin{array}{r}, 932 \\
, 000 \\
12\end{array}$ & \\
\hline & FOM & $\begin{array}{l}\mathrm{r}_{\mathrm{S}} \\
P \\
\mathrm{~N}\end{array}$ & & & & & & $\begin{array}{r}, 360 \\
, 251 \\
12\end{array}$ \\
\hline
\end{tabular}

Figure S2. Correlations between free and protein-bound glycated amino acids in malt samples. Spearman's rank correlation coefficients $\left(r_{S}\right)$ and significance of correlations were calculated using the software PASW Statistics 18. FL, fructosyllysine; FOM, formyline; MAL, maltosine; MG-H1, methylglyoxal-derived hydroimidazolone 1; ML, maltulosyllysine; PYR, pyrraline. 